\title{
LA RED VIARIA EN EL ÁREA METROPOLITANA DE ALICANTE-ELCHE
}

\author{
Alfredo Morales Gil
}

\section{INTRODUCCIÓN}

Cuando se intenta realizar la planificación del desarrollo socioeconómico del área metropolitana de Alicante-Elche debe ocupar un lugar destacado en la organización espacial el estudio de la red viaria y de los transportes con los que este área va a contar en el futuro, puesto que su porvenir tanto industrial y comercial como agrícola va a depender de las condiciones óptimas que la red actual y prevista le ofrezca. Siendo necesario el estudio de la relación que ha tenido, y tiene la política de transportes con el modelo territorial que se ha ido conformando y que existe actualmente.

El modo de producción de libre cambio consolidó un modelo territorial específico que se caracterizaría, según Fernández Duran', por los siguientes aspectos:

$1^{\circ}$ Orientación del crecimiento hacia las grandes áreas metropolitanas, como resultados de la concentración en las mismas de la población y de empleo.

$2^{\circ}$ Desertización del resto del territorio, como resultado de los fuertes [52] movimientos poblacionales que implica el proceso anterior (no es el caso de Alicante-Elche en las tierras comarcales).

$3^{\circ}$ Jerarquización del crecimiento de las áreas metropolitanas dependiendo del papel que juega cada país y cada región en el conjunto mundial. Puesto que el aparato económico local está cada vez más sometido a la estrategia internacional del capital.

Las formas de producción y consumo en grandes unidades, alta división y especialización del trabajo, gran necesidad de actividades del terciario van a ser las necesidades del capital en la fase actual de desarrollo de la red viaria del área metropolitana de Alicante-Elche, de ahí que el capital se oriente hacia esta aglomeración urbana y se revalorice más localizándose en la misma. El estado también ayuda y potencia la configuración de este modelo territorial al crear las infraestructuras y los servicios que posibiliten su funcionamiento (aunque en el caso que nos ocupa no haya sido esto totalmente cierto).

Pero la estructura metropolitana que se origina en estas grandes aglomeraciones es tremendamente desequilibrada, como consecuencia de las distintas lógicas de localización espacial de las diferentes actividades urbanas, de la existencia de la propiedad del suelo, y de una estructura social basada en clases sociales que genera y potencia la segregación espacial de los distintos sectores sociales.

\footnotetext{
${ }^{1}$ FERNÁNDEZ DURÁN, Ramón: «La quiebra de la política de transportes». I.C.E. Abril 1981, pág. 37 y siguientes.
} 
Partiendo de este planteamiento podemos afirmar, como indica Fernández Durán, que tanto en Elche como en Alicante en función de la viabilidad de estos núcleos se observa lo siguiente:

$1^{\circ}$ La concentración creciente del terciario en las áreas centrales, y en especial el terciario de carácter decisional.

$2^{\circ}$ Localización industrial en áreas periféricas.

$3^{\text {o }}$ Segregación espacial cada vez más acusada de la población de acuerdo con sus niveles de renta.

$4^{\text {o }}$ Segregación y concentración cada día más intensa de las distintas actividades urbanas (hospitales y centros comerciales).

Este modelo territorial que se ha configurado en el área metropolitana de Alicante-Elche se va a caracterizar por las elevadas exigencias de transporte que conlleva. De esta forma estas altas necesidades de transporte son consecuencia tanto de las propias características del modelo territorial como de las peculiaridades del sistema productivo, basado en la especialización espacial y no en la autosuficiencia.

Por otro lado, a nivel metropolitano se originan elevadísimas necesidades de transporte, como resultado del incremento del número de viajes motorizados y de la longitud de los mismos: igualmente se produce su concentración en el tiempo, lo que obliga a diseñar la capacidad del sistema de transporte para la hora punta, (que es lo que está intentando [53] solucionar el Ayuntamiento de Alicante, pero que no lo consigue, por toda la serie de causas que después apuntaremos) elevando considerablemente los costes de explotación o de funcionamiento. Estas necesidades de transporte se incrementan normalmente, si se intenta dar solución a las mismas mediante la utilización del vehículo privado (que es lo que ocurre en el área metropolitana de Alicante-Elche por iniciativa privada).

De lo anterior se deduce que el transporte y la red viaria son unos elementos claves de los modelos territoriales metropolitanos siendo necesarias importantes inversiones en los sistemas de transporte para satisfacer los fuertes volúmenes de tráfico que provoca este modelo, así como elevadas subvenciones públicas para hacer viable el funcionamiento de los transporte públicos.

Según todo lo anteriormente dicho en el área metropolitana de Alicante-Elche se tendrían que haber realizado las siguientes actuaciones:

$1^{\mathrm{o}}$ Potenciación de los ejes de comunicación, o de transporte que unen los núcleos urbanos principales, al tiempo que se favorecería una accesibilidad más homogénea que hubiese beneficiado al conjunto del territorio.

$2^{\circ}$ Creación de un sistema de transporte urbano que permitiera su funcionamiento y posibilitaría su crecimiento (basado en una red viaria de trama ortogonal).

Política de transporte que ni siquiera se ha realizado, a lo sumo se ha insinuado mejorando algunos de los accesos a Alicante o las obras de ferrocarril a su paso por Elche, constituyendo solamente una retahíla de buenas intenciones que desde hace 14 o 15 años se vienen exponiendo en los Planes de Ordenación Urbana y que la prensa airea de vez en cuando para que los sufridos ciudadanos no desesperen de ver hechas realidad todas esas promesas algún día.

¿Cómo se ha llegado a esta situación?, pues en primer lugar habría que señalar la potenciación y la prioridad dada al transporte por carretera, y en especial, al vehículo privado, en detrimento de otros medios de transporte, política que ha sido debida, no a la mayor o menor eficacia de este tipo de transporte, sino a los poderosos intereses económicos relacionados con este sector (industria del 
automóvil, del petróleo, del caucho), pero que en el caso italiano también ayudaron al desarrollar una red viaria apropiada. También ha influido el hecho de que el nuevo estatus socioeconómico que se consiguió en las décadas de los 60 y 70 con elementos tales de segundas residencias contribuyeran a un desarrollo mayor de la utilización del vehículo privado.

Todo esto ha implicado la construcción de importantes redes de [54] autovías y autopistas interurbanas e incluso de autopistas urbanas siguiendo la lógica del modelo territorial que se estaba creando, como son los casos de los modelos catalán y vasco, pero que en el área metropolitana de Alicante-Elche sólo se ha visto afectado en su parte negativa, ya que continuamos prácticamente con la misma red viaria de principios de siglo.

Desde 1973 a nivel mundial y desde 1975 o 1977 a nivel español este modelo entra claramente en crisis (se disparan los precios de la energía y de los vehículos), afectando fundamentalmente al transporte por carretera, base del modelo AlicanteElche sin que se pueda arbitrar otro sistema que lo sustituya puesto que el espacio que han ocupado las dos ciudades y sus zonas de residencia no se puede abandonar o reducir, por lo que el transporte se tiene que adaptar a esta estructura con el encarecimiento que supone y que por mucho que las entidades locales quieran mejorar siempre lo tendrán que hacer soportando grandes pérdidas.

Para salir de esta crisis los países occidentales más desarrollados han realizado grandes transformaciones en sus políticas territoriales y de transporte:

$1^{\circ}$ Se ha intentado detener el crecimiento de las grandes ciudades, $2^{\circ}$ frenando los programas de autopistas, $3^{\circ}$ introduciendo limitaciones al uso del vehículo privado, $4^{\circ}$ potenciación del transporte ferroviario, y $5^{\circ}$ procurando potenciar aquellos medios de transporte más eficientes desde el punto de visto energético y de la contaminación. Sin embargo el caso del área metropolitana Alicante-Elche es distinto, puesto que todas estas medidas son viables allí donde el modelo territorial llegó a funcionar, pero en nuestro caso no sucedió nada más que en su parte negativa, por ello aunque no nos guste tendremos que acceder a la realización de algunas mejoras para que sea viable la organización espacial que hoy disfrutamos, pese a la oposición de algunos grupos.

\section{LA RED VIARIA}

La red viaria de la provincia de Alicante contaba desde la prehistoria con tres rutas fundamentales las cuales discurrían por áreas geomorfológicamente deprimidas que facilitan el tránsito: la Depresión del Bajo Segura, paso natural que, desde el norte de Almería y a través del corrector del Guadalentín, venía a desembocar en la costa en las proximidades de Alicante; el Valle del Vinalopó, gran fosa que con una disposición N-S confluye sobre el pasillo anterior, justo a la altura de Elche, y finalmente los valles corredores del NW de la provincia que sirven de enlace entre la parte meridional de la provincia de Valencia y el norte de [55] la de Murcia. Como se ve, a grandes rasgos, se trata de una trama formada por dos ejes paralelos cortados por otro transversal que presentaba sus puntos de intersección donde en la actualidad se asientan las localidades de Elche y Villena, ciudades de tan remoto pasado cultural.

Estos pasos naturales se van a potenciar con el transcurso de los tiempos, y de hecho los dos primeros en la actualidad se hallan aprovechados por importantes carreteras y ferrocarriles. Así la Carretera Nacional 340 (Puerto Lumbreras-Alicante) 
y el ferrocarril Murcia-Alicante utilizan la Depresión del Bajo Segura y de ahí su fácil trazado y viabilidad. El Valle del Vinalopó está claramente recorrido desde Villena hasta Novelda por la C. N. 330 (Alicante-Almansa, localidad última donde enlaza con la 430 Albacete-Valencia) y el ferrocarril Alicante-Madrid. En Novelda este eje ve interrumpir su prolongación hasta Elche, que es hasta donde debía continuarse; la presencia del puerto de Alicante a unos $20 \mathrm{~km}$. determinó que estas importantes vías de comunicación describiesen aquí un giro hacia oriente, al ser considerado dicho puerto durante bastante tiempo como la salida más cercana de Madrid al mar.

Sin embargo, si se realiza el modelo de simulación de la red viaria de esta parte de la provincia, se ve rápidamente que existen unas rutas naturales que van a canalizar el transporte, en más de un 80 o $90 \%$ coincidente con la red real y que además insinúa trazados de vías inexistentes y cuya construcción se podría acometer con éxito económico asegurado. El resultado de esta simulación es la existencia de dos centros de primer orden, Alicante y Elche, con un gran poder de interacción del último en función de su emplazamiento por localizarse en la confluencia de dos vías naturales -Depresión del Bajo Segura y Valle del Vinalopó-, por lo que se ve claramente cómo el ser encrucijada de caminos ha permitido, en parte, facilitar su desarrollo económico ${ }^{2}$. Si se tienen en cuenta los índices de potencial de interacción entre estos dos grandes núcleos, resulta que el área que se relaciona con Elche dependería potencialmente de Alicante a través de aquel núcleo, ya que el último atrae al primero por contar con mayor número de habitantes y servicios a pesar del mal estado de su puerto, que en los últimos años ha venido debilitando su movimiento.

Con los resultados del potencial de interacción se puede intentar construir un modelo de simulación viaria terrestre. Resultaría una red simulada con un eje principal que atraviesa toda la Fosa Intrabética y que desde Alicante se prolonga hasta Benidorm, enlazando las dos grandes [56] áreas metropolitanas de Murcia y Elche-Alicante. Destaca otro eje simulado que desde Elche sigue por todo el Valle del Vinalopó hasta Villena: se trata de otra ruta perpendicular a la primera, que sirve de acceso a la Meseta y que presenta el problema de que en la práctica el trayecto Novelda-Elche de este eje simulado no ha sido potenciado lo suficiente; como ya se ha dicho, al llegar la carretera y el ferrocarril a esta primera localidad, las grandes vías se apartan del eje teórico para acercarse al puerto de Alicante. De todas formas la carretera que sigue este tramo del Valle del Vinalopó hoy día se está promocionando, aunque no alcanza la intensidad de tráfico que tiene el desvío que se dirige a Alicante.

Las carreteras.- En la actualidad el estado en que se encuentran las carreteras es muy variado. Así, la gran ruta que sigue la parte oriental de la Fosa Intrabética, materializada en la Carretera Nacional 340 desde Puerto Lumbreras a Alicante, se continúa desde aquí hacia Benidorm por la C. N. 332. Esta última, que pone en comunicación Alicante con Valencia y la frontera francesa, fue incluida en el Plan de Modernización de Carreteras REDIA, lo que la ha convertido en una aceptable ruta. Sin embargo, la C. N. 340 no ha sido modernizada, pese a que presenta una intensidad de tráfico semejante a la anterior -20.000 vehículos diarios- y une dos grandes áreas metropolitanas. Este abandono todavía es mucho más manifiesto si se

\footnotetext{
2 MORALES GII, A.: «Las comunicaciones en Murcia ante el desarrollo económico regional». Papeles del Departamento de Geografía. Universidad de Murcia, 1974, pág. 126.
} 
tiene en cuenta que es el único eje con que cuenta el área metropolitana AlicanteElche-Crevillente, donde las intensidades de tráfico diario son superiores a los 25.000 vehículos, máxime si pensamos que esta carretera a su paso por Elche y en un trayecto superior a los $10 \mathrm{~km}$. es como una calle de esta ciudad, en cuyas orillas se asientan todos los establecimientos industriales, con el tráfico que a corta distancia se engendra y del que no existen más que estimaciones aproximativas. En definitiva se trata del principal eje de comunicación que atraviesa la comarca, con una sobresaturación de tráfico y en cuyo recorrido de unos $30 \mathrm{~km}$. (Alicante-Crevillente) normalmente en los días laborables se invierte por término medio de 45 a 50 minutos, lo cual indica que la velocidad media que se puede alcanzar es de alrededor de los 35 o $40 \mathrm{~km} / \mathrm{h}$.

La ruta sobre el Vinalopó se materializa entre Novelda y Villena en la Carretera Nacional 330 de Alicante a Almansa, en donde se une a la C. N. de Albacete a Valencia, y el tramo Novelda-Aspe-Elche, que es una carretera comarcal de gran tráfico. La primera también ha sido recientemente modernizada por estar incluida dentro del Plan REDIA e igualmente ha sido mejorada la comarcal AspeElche. Además se trata de una ruta que presenta intensidades de alrededor de los 15.000 vehículos en el tramo de carretera nacional, cuyo flujo va decreciendo conforme se avanza hacia el interior, mientras que en el tramo comarcal [57]

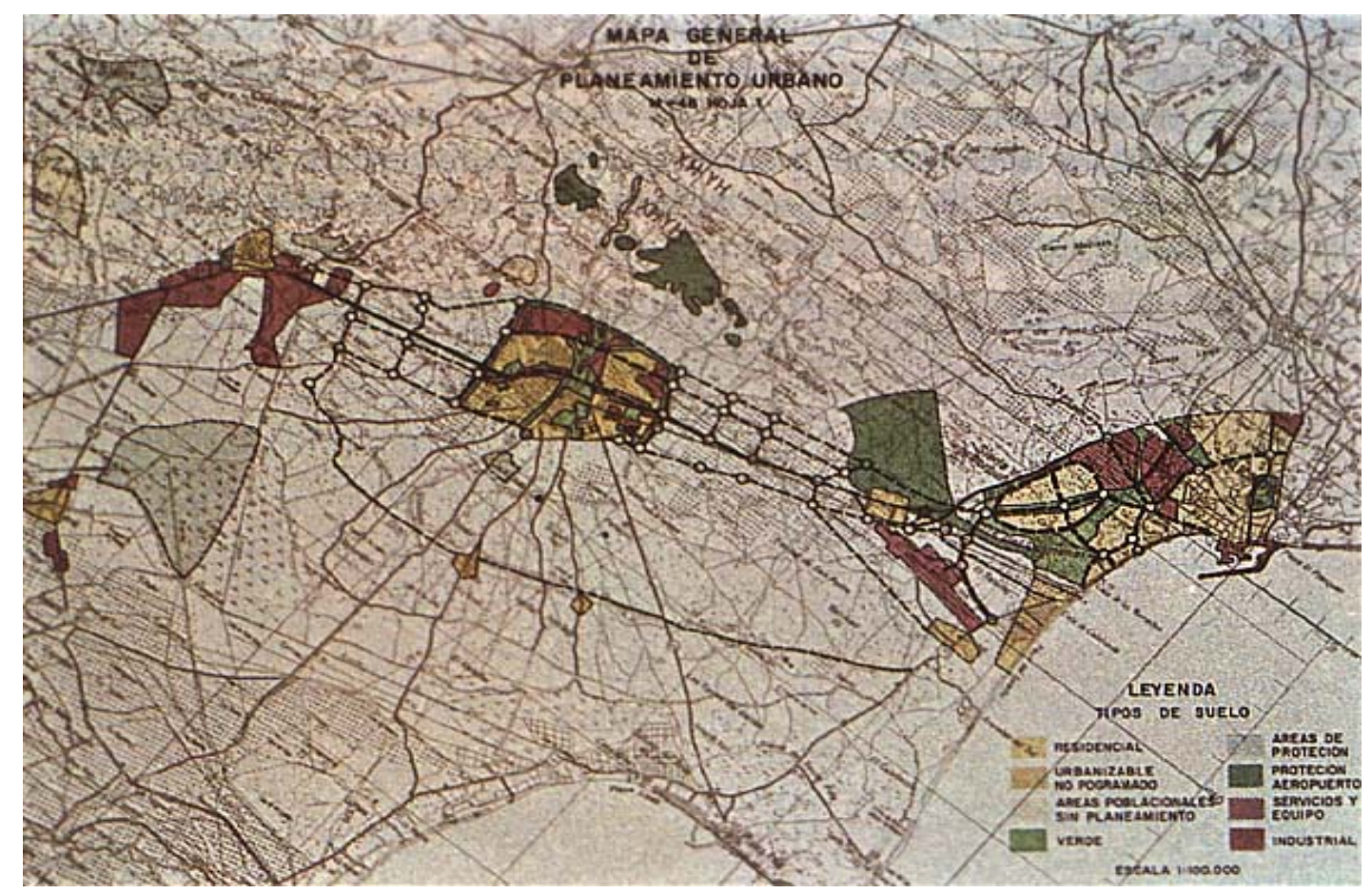

Tipos de suelo urbano en Alicante, Elche y Crevillente y red viaria prevista.

su media diaria es inferior a los 5.000. Por lo tanto son unas vías que aún no están saturadas y que solamente presentan alguna conflictividad en el trayecto Elche-Aspe, por transcurrir por una zona topográficamente accidentada, en donde, a pesar de los arreglos realizados, todavía no se ha roto con el viejo trazado caminero que la condiciona.

Del examen de la red viaria actual se deduce que el área metropolitana de Alicante-Elche tiene planteado un grave problema de cara a su desarrollo futuro por 
la necesidad urgente de otras vías terrestres que unan más rápidamente a Alicante y Crevillente, para lo cual es necesario construir otros accesos paralelos al actualmente existente, más que sobresaturado. Con este fin se han proyectado una serie de soluciones, entre ellas la de la Autopista del Mediterráneo, que seguidamente serán examinadas:

La Autopista del Mediterráneo, en su tramo Alicante-Elche, estaba prevista en el Plan de Ordenación Urbana de Elche para que entrase en servicio en 1975 y sin embargo en la actualidad todavía no se ha subastado e incluso se duda sobre su posible construcción. En aquel plan se especulaba con la posibilidad de que fuese considerada como de enlace urbano, libre de peaje, sirviendo al área urbana y «metrópolis de equilibrio» de Alicante-Elche y municipios de su zona, ya que la proximidad [58] de las dos ciudades no garantizaba el uso de la autopista de peaje para tráficos con origen y destino en estas localidades. Esto planteó la cuestión de que también se tenía que construir una autovía urbana que sirviera a estos núcleos de población congestionados, tanto para disuadir al tráfico local de usar la autopista, como para ofrecer una jerarquía coherente de tráficos.

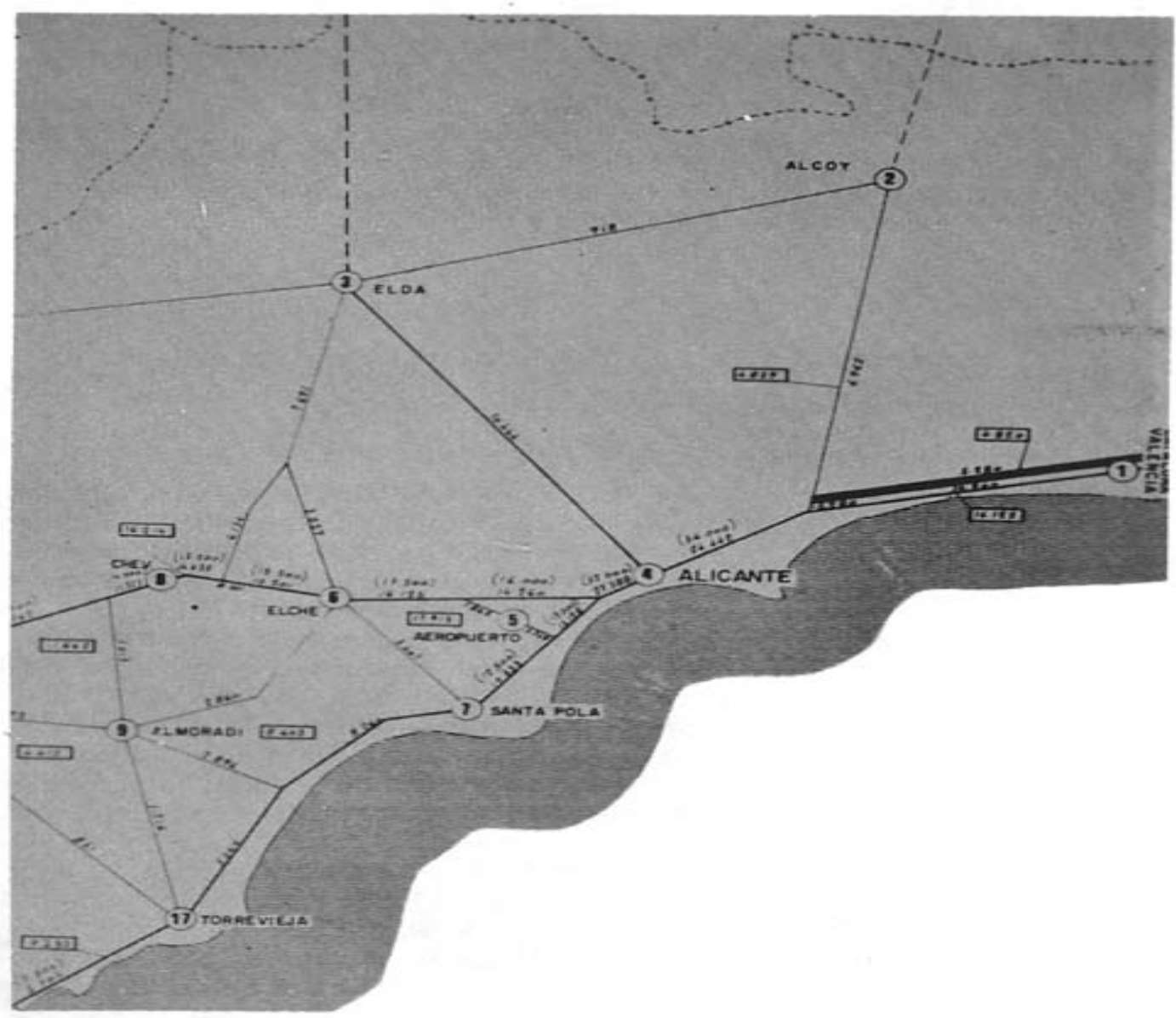

Intensidad de tráfico en 1981.

[59]

De manera que el P.G.O.U. de Elche propone la construcción de dicha autovía dando varias soluciones alternantes de trazados, pero inclinándose por el del norte, 
que arrancaría en Alicante en la prolongación de la Vía Parque, proyectada en el Plan General de 1971, pasando por la parte septentrional de la ciudad de Elche y junto a la Autopista del Mediterráneo y por debajo de ella buscar la Carretera Nacional 340 antes [60] de Crevillente, para desde aquí desviarse por el sur de dicha ciudad.

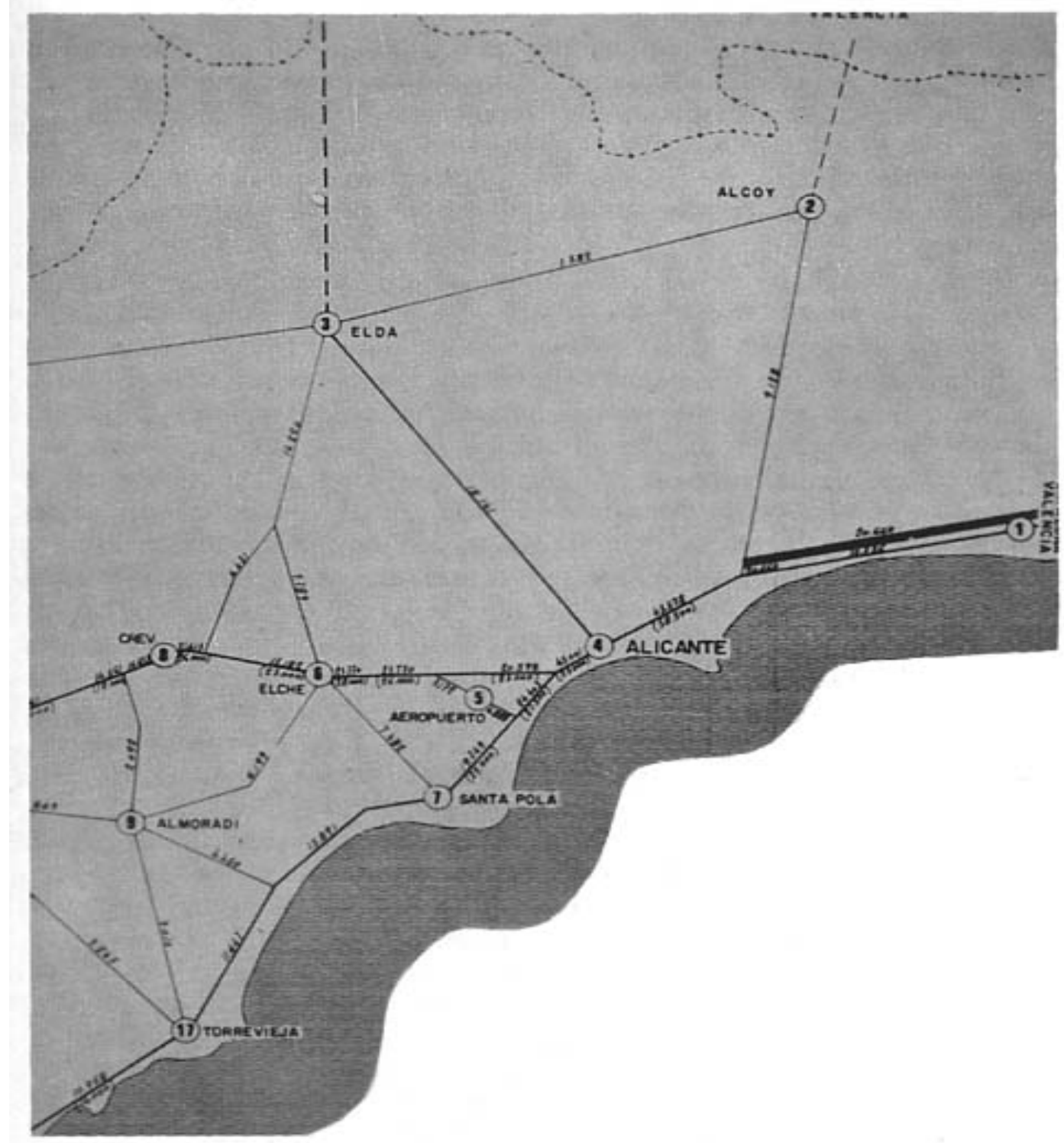

Intensidad de tráfico prevista para 1990.

Esta solución implicaría: $1^{\circ}$ Que las funciones atendidas por la autopista y la autovía serían suplementarias en su inmediata proximidad espacial por el rápido paso de una a otra en progresión jerárquica de velocidad, áreas servidas útiles y accesos sucesivos. $2^{\circ}$ Debería considerarse la autovía comarcal como una desviación de importancia, o una suplementación de la C. N. 340, ya que ésta quedaría absorbida por la red arterial de Elche y Alicante, debido a que el centro de gravedad de las dos ciudades podría situarse sobre la autopista, por lo que sería más lógico acercar dicha autovía a la autopista y evitar así rodeos innecesarios. Y La ubicación de grandes polígonos industriales se efectúa fundamentalmente al norte de la prolongación 
teórica de Alicante-Elche, por lo que era más lógico servirlos en estos cortos trayectos con una autovía.

A más corto plazo se preveía la ampliación y consolidación de la C. N. 340 a su paso por el municipio de Elche, pues normalmente es el tramo de mayor congestión. Esta carretera pasaría por el de Torrellano, de manera que cruzando la vía del ferrocarril permitiera una desviación hacia l'Altet y los Arenales y otra hacia el Aeropuerto enlazando con la actual y finalmente desde aquí ir a buscar la N. 340 en el km. 72. También de Torrellano saldría otro ramal hacia el norte que fuese a enlazar con la actual carretera local que bordea Alicante por el N, carretera hacia las Atalayas y la N. 330, de tal manera que se llegase a Alicante por dos vías divergentes, descongestionando la actual N. 332 de la costa a la entrada a la ciudad.

De todas estas previsiones de mejoras de la red viaria comarcal, proyectadas en 1972 y que, según los cálculos, algunas entrarían en servicio en 1975, no se ha realizado ninguna, ni siquiera la última mencionada. Solamente en los días de mayor tráfico de verano se realizan como solución de emergencia desvíos forzosos de entrada a Alicante por la estrecha carretera del Bacarot, que une a la N. 340 con la N. 330, que utiliza parte del antiguo trazado del tranvía de Alicante a Crevillente. Y sin embargo el incremento de tráfico en este eje tan vital para el desarrollo del área metropolitana alicantina ha ido en aumento, con unas medias en 1980 de 32.000 vehículos diarios de entrada a Alicante y de 19.000 entre Torrellano y Elche, disminuyendo ligeramente a 18.000 en el tramo Crevillente-Elche (en estos datos no se contabilizan los vehículos de dos ruedas). Nos encontramos, pues, con que se han superado con creces las posibilidades de una carretera de tipo REDIA (15.000 vehículos de intensidad media diaria) y por supuesto que en las proximidades de Elche se rebasan esos 25.000 a que se aludía con anterioridad. Se ha producido una gran congestión que se manifiesta en [61] unas grandes pérdidas de tiempo, puesto que cada día la velocidad media del flujo en el trayecto Alicante-Elche-Crevillente se ve más rebajada, con la incidencia negativa que implica. Situación que se agravará en los años próximos si se producen las previsiones que hay para el año 1990, de 45.000 vehículos diarios entre Elche y Alicante $^{3}$.

\section{ACCESIBILIDAD Y VIABILIDAD DE ELCHE}

Los accesos de la ciudad y su viabilidad interior constituyen un problema de difícil solución por sus estrechamientos y elevados aforos. La preocupación viaria ha sido una constante que se ha dejado sentir desde mediados del pasado siglo, cuando se fueron construyendo diversas carreteras y ferrocarriles que cruzaban la ciudad y el término de Elche, sobre todo en sentido Oeste-Este, que han sido analizadas en su evolución en varios trabajos ${ }^{4}$.

Elche tiene su red urbana articulada en un eje principal a lo largo del cual se polariza todo el movimiento de vehículos por el interior de la ciudad, y algunos ejes secundarios. Esta gran intensidad de tránsito lógicamente tiene unos efectos negativos bastante notables: $1^{\circ}$ Se registran en estas calles los mayores índices de

\footnotetext{
${ }^{3}$ CAAM: Estudio informativo de la Autopista del Mediterráneo. Itinerario Alicante-Murcia. Alicante 1979. Tomo 3.

${ }^{4}$ GOZÁlVEZ PÉREZ, V.: El Bajo Vinalopó. Aspectos de Geografía Humana. Valencia, Depart. de Geografia, 1975, La Ciudad de Elche. Estudio geográfico. Valencia, Dpto. Geografía 1976, pp.

ROSSELLO VERGER, V. y otros: Estudio socio-económico de Elche y su comarca. Valencia, Manuscrito 1979, pp. 487-488.
} 
contaminación; $2^{\circ}$ no se respetan las normas que exigen una separación mínima de 100 metros desde las viviendas a las calzadas de gran intensidad de tráfico y que por lo tanto generan índices de elevado número de decibelios que afectan a las personas que las habitan; $3^{\circ}$ se trata del centro comercial, donde el movimiento peatonal es muy grande por lo que todos los viandantes se ven continuamente amenazados por los vehículos que circulan por estas calles cuyas aceras tienen menos de un metro de anchas, y $4^{\circ}$ la media horaria de tráfico de 8 a 20 horas llega a ser de unos 1.100 vehículos ${ }^{5}$.

La viabilidad interior se mejorará cuando se pongan en práctica alguna de las obras -autovías, autopista, avenida de la Libertad- que están proyectadas ${ }^{6}$. En el Plan de 1972 se intentaba organizar una red [62] viaria ortogonal con el fin de dejar el

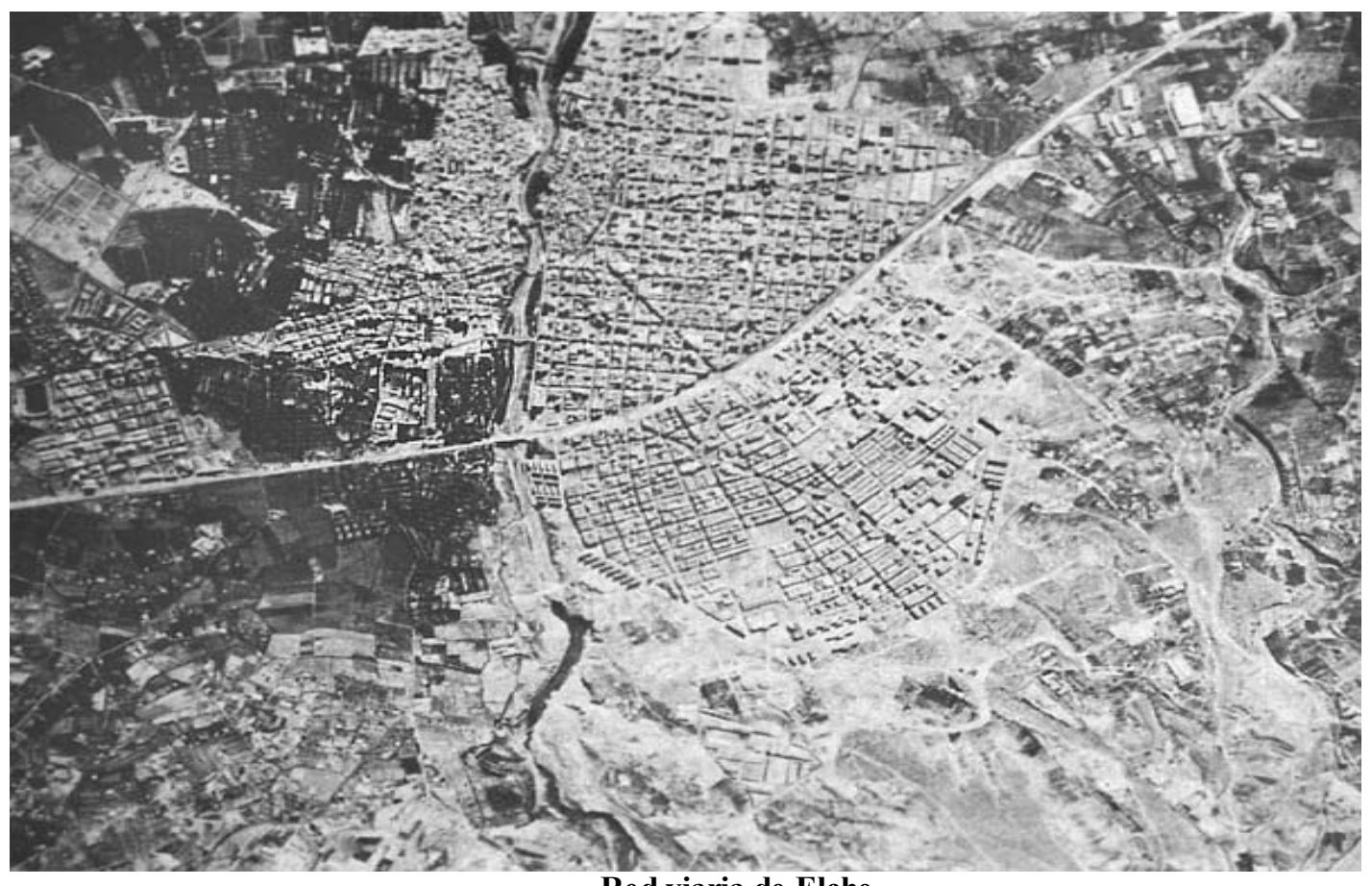

Red viaria de Elche.

núcleo histórico de la ciudad con muy poco tráfico. Este planteamiento incide en la conservación y valoración del núcleo antiguo, especialmente la Vila Murada, como una amplia zona peatonal con calles totalmente libres de tráfico, con una rehabilitación cultural de calles y plazas, que en la actualidad no existe por estar todo invadido por el automóvil. Esta área coincide también con la comercial y por lo tanto esta actividad se vería igualmente beneficiada de la tranquilidad que gozaría y para que su vida no se viera afectada por los inconvenientes de desabastecimiento se tendrían que delimitar unas horas para realizar la distribución de mercancías y unos puntos reservados para la penetración de vehículos ligeros que atendiesen sus necesidades. En cuanto al problema que los comerciantes argumentan de que si se suprime la circulación rodada, el público dejaría de frecuentarla, se resolvería mediante la creación de una orla de aparcamientos subterráneos en las plazas y huecos del Norte de la Vila Murada y flanco oriental del Raval. Así se podía crear un

\footnotetext{
${ }^{5}$ ROSSELLO VERGER, V. y otros: Obra Citada, pp. 488.

${ }^{6}$ EXCMO. AYUNTAMIENTO DE ELCHE: Anteproyecto de Urbanización de la Avinguda de la Llibertat, Elche, 1980, sin paginar.
} 
centro peatonal en torno a la Corredora en su trayecto Plaza Mayor-Glorieta. Proyecto que por desgracia todavía no ha sido realizado. [63]

\section{ACCESIBILIDAD Y VIABILIDAD DE ALICANTE}

Las causas que explican la caótica situación que se origina en determinadas horas punta en la circulación interior y en la canalización del tráfico de las vías que penetran en la ciudad de Alicante pueden ser las siguientes:

I. Las carreteras nacionales que confluyen en Alicante, así como alguna comarcal -la de Castalla-, lo hacen con una disposición radial convergente sobre el centro comercial sin que exista ninguna vía de circunvalación que las una y sobre todo que proceda a una repartición del tráfico que va a la ciudad antes de llegar al centro, todo lo cual provoca en primer lugar un efecto de cuello de botella a la entrada que se deja sentir a veces en varios kilómetros, a pesar de las mejoras de desdoblamiento de calzada que en algunas de estas carreteras se han hecho como en el caso de las salidas hacia Valencia, Madrid y Murcia. En segundo lugar habría que indicar que a ambos lados de estos accesos se han producido localizaciones industriales, comerciales y a veces barrios dormitorios que generan movimientos pendulares de corto recorrido pero que contribuyen a acentuar todavía más esa situación de sobresaturación de tráficos que padecen dichas vías.

II. Si antes se indicaba que las vías convergentes lo hacían sobre el núcleo comercial de la ciudad, todavía hay que acentuar más esta situación poco propicia para una buena viabilidad interior, que viene condicionada por la ausencia de grandes arterias que crucen de W. a E. el centro urbano y que sean capaces de absorber todo el tráfico que las carreteras nacionales vierten sobre ella, además del movimiento interior de vehículos que ella misma genera. Así sucede que el único eje capaz de realizar esta misión era el que bordea a la población por su parte meridional en contacto con el mar, por lo que se constituyó con el tiempo en la vía que más tráfico soportaba, con intensidades que hoy día rondan los 30.000 vehículos de media diaria ${ }^{7}$. Pero esta carretera-calle se ha visto continuamente disminuida en sus posibilidades por varias razones: $1^{\circ}$ Se ha producido una pugna entre la ciudad que busca por aquí una zona de esparcimiento en contacto con el mar -playa del Postiguet, Explanada y puerto deportivo- y la función de tránsito, en beneficio de la primera, de forma que en los tres últimos años ha ido perdiendo una de sus calzadas para convertirla en zona peatonal, por lo que el tráfico se ha tenido que concentrar en la que corre bordeando el puerto con los consiguientes problemas de sobresaturación, muy especialmente [64] de primavera a otoño en que el atractivo de la playa hace confluir a ella más tráfico. $2^{\circ}$ Sobre esta vía se acumula la circulación que generan los dos accesos al puerto, con gran porcentaje de vehículos pesados, entre ellos camiones de cemento procedentes de la fábrica de San Vicente, que en ocasiones permanecen operando las 24 horas del día, con una incidencia de ruido considerable sobre esta zona residencial y de esparcimiento. Y otra cuestión que gravita sobre este eje y que lo hace más conflictivo es el hecho de que por él crucen todos los transportes de mercancías peligrosas que circulan por la nacional 340, como son los camiones que arrastran cisternas de gases licuados, por no existir ninguna circunvalación que lo evitara. Todo ello convierte este eje en el más problemático de toda la ciudad, cuya descongestión es cada día más apremiante, para que de esta

\footnotetext{
${ }^{7}$ CAAM: Obra citada. Tomo 3, sin paginar.
} 
forma la población vuelva a recuperar su área de encuentro y recreo por excelencia, que fue la que le dio prestigio y atractivo en la primera mitad de siglo.

III. La disposición radial de las carreteras que convergen sobre el centro de la ciudad, sin una vía de circunvalación que las una por la periferia del casco urbano, no sólo ha provocado una concentración excesiva de tráfico en el centro, como queda indicado, sino que este hecho ha tenido una repercusión muy grande en el desarrollo

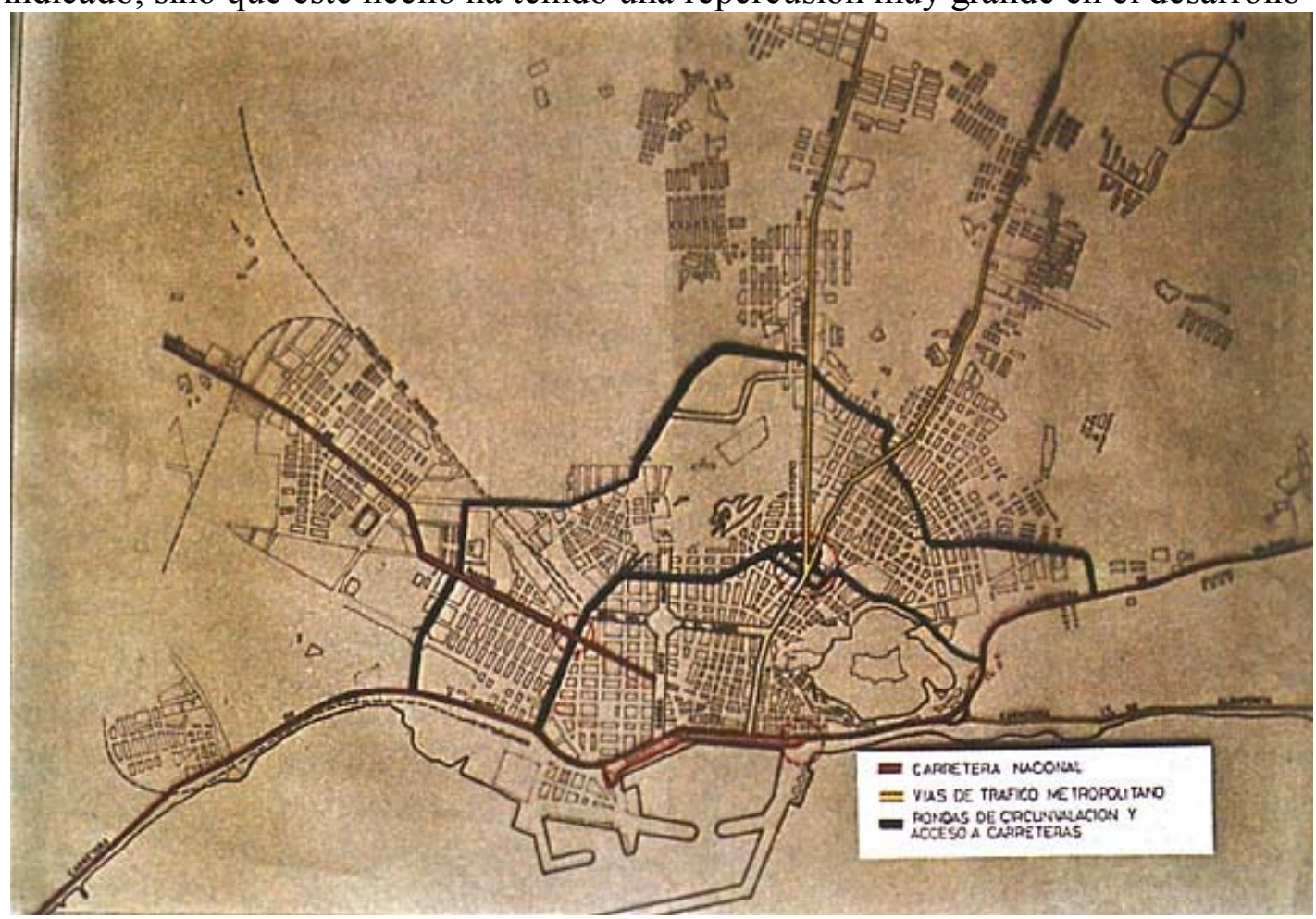

Principales arterias y puntos conflictivos del tráfico de Alicante.

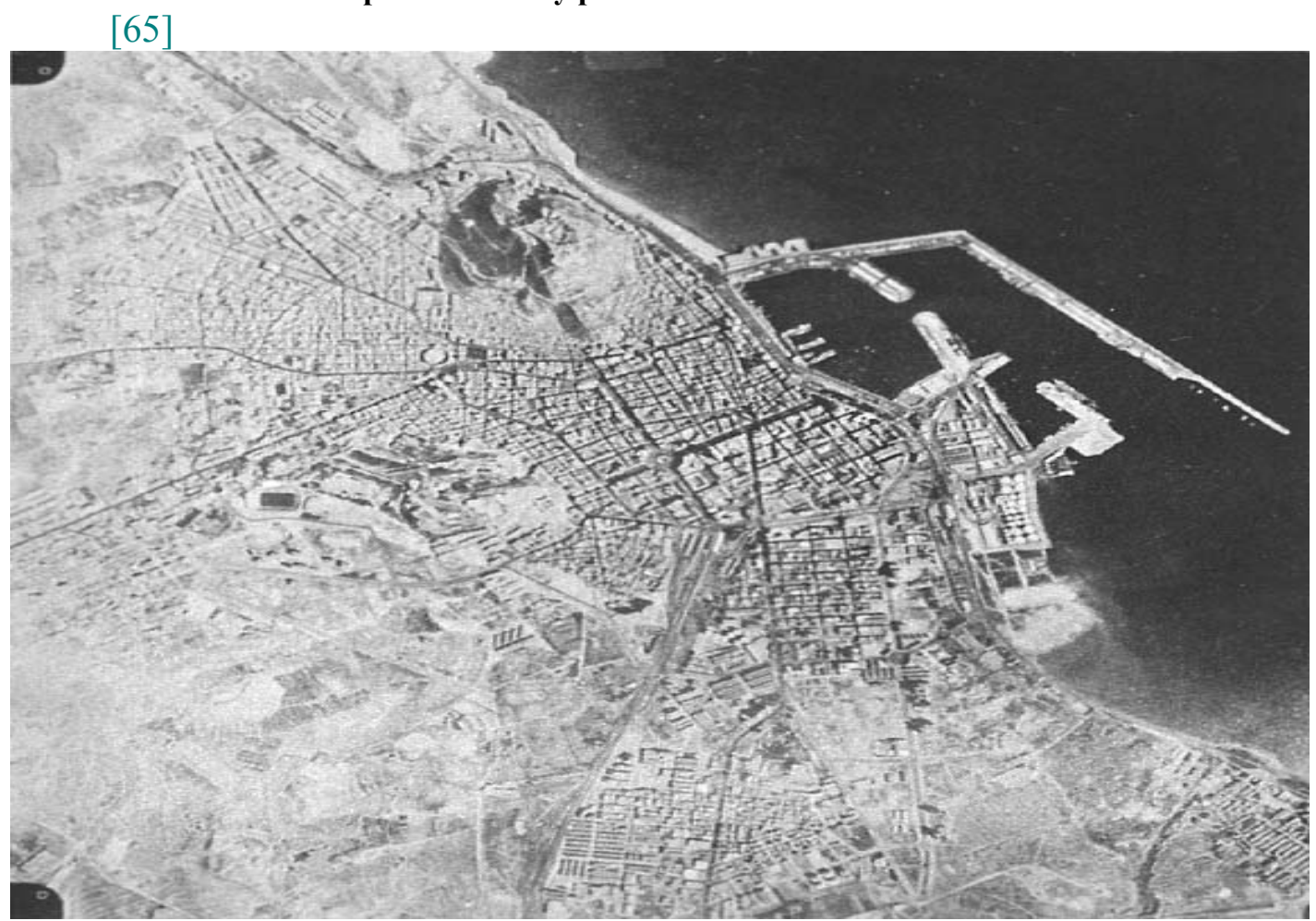

Red viaria de Alicante. 
urbanístico producido en los últimos veinticinco años, que lo ha hecho de una forma tentacular siguiendo estas arterias y dejando espacios vacíos entre ellas, lo que da al pleno de la ciudad el aspecto de una media estrella adosada a la costa.

Lógicamente esta ordenación urbanística ha influido en la viabilidad interior del casco urbano alicantino, que se manifiesta en los siguientes aspectos: a) Concentración de tráfico en las calles de Pérez Galdós, Alfonso El Sabio, Dr. Gadea y Explanada, creando varios puntos conflictivos, como las inmediaciones de la Plaza de los Toros, extremo oriental de la Explanada, convergencia de Maisonnave con la Carretera de Madrid, Óscar Esplá y Av. de Salamanca, todos los cuales en días de mucho movimiento pueden verse saturados y de esta forma dificultar la [66] viabilidad interna de la ciudad. b) Al no existir eje de circunvalación, las comunicaciones entre los barrios periféricos se tienen que hacer pasando obligatoriamente por el centro; así, por ejemplo, para desplazarse de La Florida a la Residencia Sanitaria 20 de noviembre hay que desviarse al centro, pues aunque existen unas calles que por el estadio Rico Pérez podrían comunicar ambos barrios, éstas adolecen del carácter de vías de circunvalación y tienen que salvar la barrera del ferrocarril, de forma que este sistema de enlace interno provoca por un lado una pérdida de tiempo en el desplazamiento, a la vez que un encarecimiento por mayor consumo de combustible tanto para el vehículo privado como para el público, y por otro lado produce una segregación espacial que también se traduce en social puesto que el centro, mejor comunicado, se revaloriza en exceso mientras que la periferia se va convirtiendo cada vez más en suburbial por el aislamiento a que se ve sometida frente al núcleo comercial, administrativo y de decisión a la hora de mejoras urbanísticas. c) La existencia de espacios vacíos entre los radios de las vías de penetración crea una zona de difícil ocupación urbanística por falta de comunicaciones, que sólo ve superada esta situación cuando existe una intencionalidad oficial, como en el caso del polígono del «Gran San Blas» que se está modificando por parte del M.O.P.U. y del Ayuntamiento al favorecer la aparición de grupos de viviendas el primero y hacer una concentración escolar el segundo, que a su vez genera más tráfico en las horas punta al no estar las escuelas en las proximidades de las viviendas, salvo excepciones.

La solución a todos estos problemas está en la construcción de una vía de circunvalación que una todas las vías de penetración en Alicante, al tiempo que permita una distribución del tráfico en varios puntos, si bien los accesos al puerto harán que el eje que en la actualidad envuelve a la ciudad por la zona costera no desaparezca totalmente, pero se vea bastante aligerado en su movimiento, especialmente de grandes camiones y del transporte de mercancías peligrosas que constantemente amenazan su tranquilidad. Este proyecto ha sido considerado de vital necesidad y urgencia por parte de las autoridades competentes desde que se redactó el «Plan de Ordenación Urbana de 1968», que preveía dos grandes realizaciones -Vía Parque y Gran Vía- como carreteras urbanas de circunvalación, además de un enlace de autopistas entre San Juan y Torrellano. La falta de acuerdo entre el M.O.P.U. y el Ayuntamiento ha sido la causa de su aplazamiento, situación que hay que salvar lo antes posible en beneficio de una mejor viabilidad interna y ordenación urbana más racional.

Departamento de Geografía Universidad de Alicante 